\title{
Letter to the Editor: Medical School Ranking and Physician Opioid Prescribing
}

$\mathrm{O}$ ur 2018 paper $^{1}$ used data on the near universe of opioid prescriptions to document the fact that physicians who completed their initial training at higher-ranked medical schools wrote significantly fewer opioid prescriptions between 2006 and 2014. Our analyses indicated that part of the relationship between medical school ranking and opioid prescribing was likely driven by differences in training. $\mathrm{Ly}^{2}$ zooms in on a sample of primary care practitioners treating elderly Medicare patients with new low back pain between 2011 and 2014 and suggests that there is no association between medical school rank and opioid prescribing in this population.

There are a number of reasons that might explain why Ly's results differ from ours. We explicitly examined three of Ly's proposed explanations-inclusion of location fixed effects, use of per patient measures, and focus on an older population - in our original paper. In particular, we documented that the relationship between opioid prescribing and medical school rank existed even among physicians in the same specialty practicing in the same hospital or clinic. Using publicly available Medicare Part D data, we further demonstrated that the relationship between opioid prescribing and initial medical school rank existed when the outcome was either prescription levels or per patient prescription rates. We have also verified that our results are insensitive to controlling for patient age, gender, and insurance type, suggesting that Ly's fourth explanation-adjustment for patient covariates - cannot account for the difference.

This suggests that the difference in findings is likely driven by differences in the sample. In contrast to the primary data used in our study, which covered nearly all opioid prescriptions in each year, Ly's data accounts for less than $0.01 \%$ of opioid prescriptions over the sample period and focusses on the age group with the lowest rates of prescription opioid misuse. $^{3}$ It may therefore be misleading to generalize Ly's findings to other clinical scenarios and broader populations that account for the vast majority of opioid prescriptions.

Ly concludes that "Medical school ranking may not be a useful proxy for identifying higher opioid-prescribing physicians." This conclusion is not supported by more comprehensive data. While there is more work to be done to understand what drives differences in prescribing, and Ly makes strides in that direction, the fact remains that policies to address prescribing should be targeted towards the providers who write the largest quantities of prescriptions - and these providers disproportionately come from lower-ranked schools.

\author{
Janet Currie, $P h D^{1,2}$ \\ Molly Schnell, $P h D^{2,3}$ \\ ${ }^{1}$ Department of Economics, Princeton University, \\ Princeton, NJ, USA \\ ${ }^{2}$ National Bureau of Economic Research, \\ Cambridge, MA, USA \\ ${ }^{3}$ Department of Economics, Northwestern University, \\ Evanston, IL, USA
}

Corresponding Author: Janet Currie, PhD; Department of Economics, Princeton University, Princeton, NJ, USA (e-mail: jcurrie@princeton. edu).

\section{Compliance with Ethical Standards:}

Conflict of Interest: The authors declare that they do not have a conflict of interest.

\section{REFERENCES}

1. Schnell, M., J. Currie. Addressing the Opioid Epidemic: Is There a Role for Physician Education? Am J Health Econ. 2018; 4: 383-410.

2. Ly D. Association Between US News and World Report Medical School Ranking and Physician Opioid Prescribing for New Low Back Pain, 20112014. J Gen Intern Med. 2019; 35: 961-964.

3. Center for Behavioral Health Statistics and Quality. 2014 National Survey on Drug Use and Health: Detailed Tables. Substance Abuse and Mental Health Services Administration, Rockville, MD. 2015.

Publisher's Note: Springer Nature remains neutral with regard to jurisdictional claims in published maps and institutional affiliations. 